\title{
Stochastic Modeling of Depth Based Routing in Underwater Sensor Networks
}

\author{
Kishor Patil ${ }^{\mathrm{a}, *}$, Mohsin Jafri ${ }^{\mathrm{b}}$, Dieter Fiems ${ }^{\mathrm{a}}$, Andrea Marin ${ }^{\mathrm{b}}$ \\ ${ }^{a}$ Ghent university, Department of Telecommunication and Information Processing, Sint-Pietersnieuwstraat 41, 9000 Ghent, Belgium \\ ${ }^{b}$ Department of Environmental Sciences, Informatics and Statistics, Università Ca' Foscari Venezia, Italy
}

\begin{abstract}
Interest in underwater wireless sensor networks (UWSNs) based on acoustic communication has rapidly grown over the last decade. In this field, the design of energy-efficient communication protocols is a crucial task as battery replacement may be unfeasible in practical scenarios. While routing protocols play a pivotal role in determining the efficiency of UWSNs, only a few studies investigate analytical stochastic models for their quantitative analysis and optimization. In this work, we consider a popular routing protocol for UWSNs, namely Depth Based Routing (DBR), and introduce a stochastic model for numerically deriving important performance indices, like the end-to-end delay, the energy consumption and the delivery probability, in terms of the configuration parameters. The model accounts for peculiar factors of UWSNs, including the impact of node deployment and mobility, and the high transmission loss of the acoustic channel. We present insights that are useful in setting DBR configuration parameters to optimize the trade-off between delivery probability, energy consumption and end-to-end delay.
\end{abstract}

Keywords: Depth-Based Routing; Stochastic modeling; Energy efficiency; Underwater sensor networks

\section{Introduction}

Over the last two decades, underwater applications like seabed management, sea-mine detection, environmental monitoring, etc. have motivated the adoption of Underwater Wireless Sensor Networks (UWSNs) as a communication infrastructure. As a consequence, many research effort has been devoted to study their performance and derive guidelines for their design. In contrast to most terrestrial wireless networks, UWSNs widely adopt acoustic communication as its intrinsic properties like low signal interference and large transmission coverage make it suitable for the underwater environment. Like their terrestrial counterparts, UWSNs adopt multi-hop routing protocols that aim at delivering the harvested data packets to on-surface sink nodes. The design of these routing protocols must account for the energy consumption of the network - battery replacement is considered unfeasible or prohibitively expensive - as well as for common performance indices like the expected end-to-end delay, the packet delivery probability and the network throughput.

Among routing protocols for UWSNs, an important role is played by localization-free protocols [1]. These assume that nodes only know their depth (and potentially that of their neighbours) when taking routing decisions. Such protocols are mostly adopted for networks with high node

\footnotetext{
*Corresponding author

Email addresses: patil.kishor@ugent.be (Kishor Patil), mohsin.jafri@unive.it (Mohsin Jafri), dieter.fiems@ugent.be (Dieter Fiems), marin@unive.it (Andrea Marin)
}

mobility, channel fading etc. as they are capable of finding new routes for each transmission. While such protocols provide a high network resilience, this may come at the expense of considerable energy consumption caused by redundant packet transmissions and the hidden terminal problem. Therefore, it is crucial that the parameters of the protocol are configured to balance the various performance indices. For a given cost, the optimal configuration achieves the best trade-off between energy consumption, mean end-to-end delay, throughput, delivery probability, etc.

One of the most widely used localization-free routing protocols is Depth Based Routing (DBR) [2]. DBR uses the depth information of nodes to build a route from the source sensor node to the on-surface sink. The nodes estimate their depth by on-board pressure sensors and add this information to any packet they send out, such that all receivers can calculate the depth difference between themselves and the transmitter. DBR adopts a receiver-based forwarding scheme in which the potential forwarders are chosen on the basis of the depth difference between the sender and the receiver. In order to reduce redundant transmissions, DBR introduces the concept of a packet holding time, i.e., a time that a potential forwarder waits before sending the packet. The holding time is inversely proportional to the depth difference between sender and receiver. For a given transmission range, this mechanism enables the protocol to cover the longest distance towards the surface at each forwarding step, as receivers further away from the surface wait longer and then drop the packet if they overhear the communication of the nodes closer to 
the surface. Fig. 1 illustrates the basic operation of DBR. The optimal configuration of DBR depends on a set of parameters, among which a pivotal importance is played by the choice of the transmission power and the holding time. Specifically, the transmission power determines the distance over which the packet can be received correctly, which in turn affects the number of hops needed to reach the sink, the overall energy consumption, the packet delivery probability and the end-to-end delay. In general, there is a trade-off between long and short distance transmissions, i.e., too many hops to the destination lead to high end-to-end delays while too few require a high transmission power that consumes the nodes' batteries too fast.

In this paper, we study this trade-off by proposing and analyzing a stochastic model for DBR. In particular, we propose a numerically tractable stochastic model that can accurately capture the dynamics of DBR. For this model, we show that one can efficiently calculate the main performance metrics including the mean end-to-end delay, the delivery probability and the expected energy consumption. In comparison to simulation, these performance measures can be calculated much faster, which in turn allows for speeding up the optimization procedure to find the optimal configuration of the UWSN. With respect to previous work that addresses the problem of assessing DBR performance, this is the first analytical model taking into account node deployment and mobility, as well as the the intrinsic properties of acoustic transmissions including the path loss and the bit error rate. The model is validated by comparing its results with the estimates obtained by resorting to stochastic simulations.

The remainder of this paper is structured as follows. In Section 2 and 3, we relate the contribution of the present paper to the literature by discussing related work and introducing the motivation for the model at hand, respectively. Section 4 then presents the stochastic model and the numerical algorithm to efficiently calculate the key performance measures of the model. In Section 5, we illustrate our approach by some numerical examples before drawing conclusions in Section 6.

\section{Related work}

In the last decade, the performance analysis of UWSNs and their optimization and control have drawn the attention of many researchers (see, e.g., [3]), simulation rather than analytical models being the primary tool to study performance. In general, while simulation models can be very accurate, obtaining performance metrics is often time consuming and their adoption for optimization purposes can be very expensive. Among the analytical models, Guan et al. [4] examine the spatial and temporal uncertainty of the underwater acoustic channel and develop a statistical model that is used to propose a novel distributed MAC scheme with an optimized transmission strategy. Pignieri et al. [5] propose an analytic model for channels in underwater networks. In these papers, neither the computation of the mean end-to-end delay and energy consumption nor the impact of the routing protocols on the networks performance is considered.

UWSNs are different in many aspects from their terrestrial counterparts. For example, energy consumption is higher due to longer distances that need to be crossed and due to complex signal processing (see, e.g., $[6,7,8]$ for some works that improve the energy consumption at the physical and MAC layers). Since the communication speed is equal to the speed of sound, the propagation delay is also much higher than the speed in the terrestrial networks. De Souze et al. [9] propose a model to analyze the energy consumption in multi-hop UWSNs. Some works study stochastic scheduling of data transmissions to deal with network latency while accounting for energy consumption. Among these, Marinakis et al. [10] formulate the channel access problem in terms of directed graphs and provide a heuristic to obtain the minimum latency. However, the acoustic absorption (as in Thorp's experimental formula [11]) and routing protocols in the computation of the transmission loss are abstracted out. Similarly, Li et al. [12] develop a new routing protocol based on a Markov model used to optimize the trade off between the packet delivery probability and the energy consumption. While the authors provide an energy-aware routing path selection, the unreliability of the links and the impact of node mobility on data transmissions are ignored. The optimality of opportunistic protocols in UWSNs is considered in [13].

In UWSNs, full localization schemes are difficult to implement since nodes consume considerable energy to access the localization services. Yu et al. [14] propose a Weighting Depth and Forwarding Area Division DBR routing protocol which accounts for the depth difference of two hops: not only the depth difference of the current hop but also the depth difference of the next expected hop. It achieves an improved packet delivery ratio as it tackles the issue of coverage holes during transmission towards the sink. More recent work from Rehman et al. [15] proposes an energy efficient cooperative opportunistic routing protocol which improves the network lifetime by applying fuzzy logic for relay node selection towards the network sink. Chao et al. [16] minimize the expected number of transmissions for successful delivery of a packet to the sink. In [17], the authors have proposed a novel routing scheme Channel Aware routing protocol (CARP) in which reliable communication between the hops is preferred on the basis of the transmission history of the nodes. Shadow zones in the network are also identified and network throughput is increased. Xie et al. [18] propose a Segmented data transport protocol (SDRT) which mainly employs block by block packet transmission. They combine FEC and ARQ to formulate their hybrid approach along with improving the channel utilization. Some other works [19, 20] exploit extra capabilities of a node, e.g., the ability to move autonomously in order to minimize the energy consumption in the deployed sensor network. 


\section{Problem Motivation and contribution}

In this paper, we consider the DBR scheme and introduce a stochastic model to analyze its performance. In contrast to previous work which relies on simulation, we provide an algorithm to efficiently calculate various performance indices, including the distribution of the number of hops it takes to send from a point other than the bottom of the network to a surface node, the level dependent energy consumption and the mean end-to-end delay. As the model accounts for the impact of node deployment and the high transmission loss of the acoustic channel, it can be used to understand the behaviour of DBR at the network level.

Although many stochastic models have been proposed and analyzed to study the characteristics of underwater channels, only few results are available for studying and optimizing routing protocols of UWSNs. We aim to fill this gap by devising a probabilistic model to assess the performance of DBR. Analytic models of UWSNs are important tools that provide insight into the dynamic behavior of the communication schemes at the physical, the MAC as well as at higher network layers. The model proposed here finds practical applications in designing a UWSN equipped with DBR. It can support the roll-out of such a UWSN by finding the optimal configuration parameters without resorting to time-expensive simulation studies. Therefore, we can summarize our contribution as follows:

- We propose a two-dimensional probabilistic model of DBR which captures its key characteristics, including transmission delays, the acoustic channel, node mobility and holding times based on depth differences.

- We show that the key performance indices can be calculated quickly. We devise computation schemes to calculate the hop-distribution, the delivery probability, the level-dependent energy consumption and the end-to-end delay.

- By means of a numerical example, we discuss how the network performance depends on environmental characteristics like the node density and the overall transmission loss, thereby showing that the model at hand can support the design of UWSNs.

\section{DBR and its Stochastic Model}

In this section, we present DBR and introduce a stochastic model to assess its performance.

\subsection{Depth based routing}

DBR [2] is a packet forwarding protocol for UWSNs which uses depth information to relay information from underwater sensors to data sinks at the surface. In DBR, every node has a pressure sensor which enables the node to estimate its depth while the nodes are in general unaware of their exact 3D position.

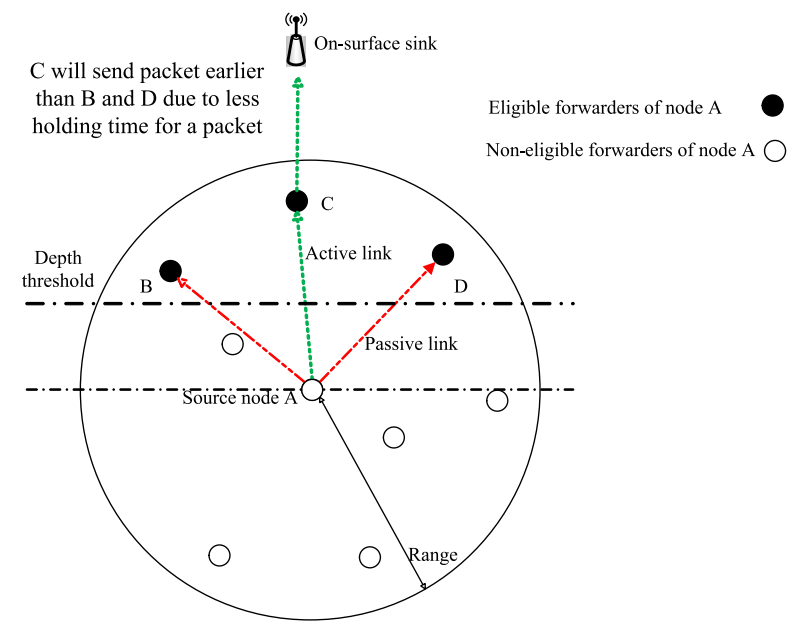

Figure 1: Methodology of DBR

The key determinant which decides which node will forward the information is the depth difference between the sender and the receiving node. More precisely, when a node transmits a packet, it includes its own depth information in the header. Among the (possible multiple) nodes that correctly receive the packet, the next forwarder is decided by two mechanisms. First, a depth threshold is installed. This is the minimum depth difference that allows a receiver node to become an eligible forwarder. Secondly, with the aim of maximizing the distance covered by one hop, a depth-difference dependent holding time is introduced. That is, every packet to be forwarded is kept at the receiver node for a time interval which decreases linearly with the depth difference between the sender (as indicated in the packet header) and itself. In this way, nodes closer to the surface have shorter holding times and actually forward the packet if they correctly receive the packet. Once a node overhears a re-transmission of a packet that is stored in its priority queue, it removes this packet and cancels its holding time in order to prevent redundant transmissions.

In accordance with [2], the holding time $D_{H}$ for a certain depth difference $d$ can be expressed as follows,

$$
D_{H}(d)=\left(\frac{2 \tau}{\delta}\right)\left(T_{r}-d\right),
$$

where $T_{r}$ is the maximal transmission range of a node, $\tau$ is the maximum propagation delay of one hop, i.e., $\tau=T_{r} / v_{0}$ where $v_{0}$ denotes the sound propagation speed in water, and $\delta$ is a scaling factor which is chosen in order to achieve optimal performance of the network and to minimize the hidden terminal problem. We choose $\delta=T_{r} / 4$ in the remainder in accordance with literature [2].

\subsection{Node location model}

For the sake of readability, we present a model for DBR in a two-dimensional environment. The extension to 3 dimensions is straightforward and summarized in section 4.7 . 


\begin{tabular}{|l|l|}
\multicolumn{2}{l}{ Table 1: List of Notations used in the model } \\
\hline Notation & Definition \\
\hline \hline$U$ & Total number of deployed nodes in the network \\
\hline$L$ & Total number of depth levels in the network for a node \\
\hline$\Delta_{d}$ & Total depth of the network \\
\hline$\Delta_{w}$ & Total width of the network \\
\hline$M$ & Number of horizontal positions on a particular level for a node \\
\hline$S$ & Horizontal position of the source node at the bottom level of the network \\
\hline$T$ & Horizontal position of the sink node at the top level of the network \\
\hline$(i, j)$ & Location of a node with position $j$ at level $i$ in the network \\
\hline$b_{j}$ & Probability that the node occupies position $j$ at a particular level in the network \\
\hline$d_{i j}^{i^{\prime} j^{\prime}}$ & Euclidean distance between nodes at locations $(i, j)$ and $\left(i^{\prime}, j^{\prime}\right)$ \\
\hline$T_{r}$ & Transmission Range of a node \\
\hline$N^{i j}$ & Set of nodes within the transmission range of node at $(i, j)$ \\
\hline$p_{m}(d)$ & Probability that the packet is successfully delivered over distance $d$ \\
\hline$P_{i j}^{i^{\prime} j^{\prime}}$ & Acceptance probability of a packet from location $(i, j)$ to $\left(i^{\prime}, j^{\prime}\right)$ \\
\hline$h_{k}(i, j)$ & Probability that packet is accepted at $(i, j)$ in $k$-hops \\
\hline$D_{P}\left(i, j ; i^{\prime}, j^{\prime}\right)$ & Propagation delay when the packet is sent from $(i, j)$ to $\left(i^{\prime}, j^{\prime}\right)$ \\
\hline$D_{H}\left(i, j ; i^{\prime}, j^{\prime}\right)$ & Holding time when the packet is sent from $(i, j)$ to $\left(i^{\prime}, j^{\prime}\right)$ \\
\hline$w_{i j}^{k}$ & Mean delay of $k$-hop communication to reach $(i, j)$ \\
\hline$c_{i j}^{k}\left(i^{\prime}, j^{\prime}\right)$ & Mean energy consumption at position $\left(i^{\prime}, j^{\prime}\right)$ from $(i, j)$ in $k$-hop communication \\
\hline
\end{tabular}

We consider an UWSN with $U$ nodes. The target or sink node is located at the surface level whereas the source node is located at the bottom. While assuming fixed positions for source and sink, we allow for movement of the nodes that relay the information. In particular, we divide the total depth difference $\Delta_{D}$ between source and sink into $L+1$ depth levels, level 0 being the level of the source and level $L$ being the level of the sink. One node is present at each depth level, we have $U=L+1$, which randomly moves in the horizontal direction.

The nodes can move, but remain at a fixed depth level. To simplify the analysis, we divide the range $\Delta_{W}$ in which the nodes move horizontally into $M$ slots with the same length, and assume that the node is always located at one of the $M+1$ slot boundaries (which we label from 0 to $M$ ). The horizontal position of the node at each level is assumed to be an independent random variable. Let $b_{j}$ denote the probability that the node is in horizontal position $j$. We here assume that the distribution of the horizontal position of the node is independent of the level $(j \in\{0,1, \ldots, M\})$. In the remainder, the notation "node $(i, j)$ " refers to the node at level $i$ and horizontal position $j$. For ease of reference, we enlist the major notations of the model in Table 1.

\subsection{Delivery probability of a node}

We consider the underwater acoustic channel that is described in [21]. The path loss $A(d, f)$ of the acoustic channel over a Euclidean distance $d$ for a signal having frequency $f$ can be expressed as,

$$
A(d, f)=d^{s} a(f)^{d} .
$$

The path loss $A(d, f)$ is expressed in decibels referenced to 1 micro-Pascal ( $\mathrm{dB}$ re $1 \mu \mathrm{Pa}$ ) where $d$ is expressed in $\mathrm{km}$ and $f$ in $\mathrm{kHz}[22]$. The spreading factor $s$ describes the geometry of the propagation; a spreading factor $s=2$ corresponds to spherical spreading while a spreading factor $s=1$ corresponds to cylindrical spreading. Finally, the absorption coefficient $a(f)$ depends on the frequency and is expressed in $\mathrm{dB} / \mathrm{km}$ using Thorp's experimental formula, see [11].

We can then express the average Signal-to-Noise ratio (SNR) over the distance $d$ in terms of the path loss,

$$
\Gamma(d)=\frac{e_{b}}{N_{0} A(d, f)}=\frac{e_{b}}{N_{0} d^{s} a(f)^{d}} .
$$

Here, $e_{b}$ and $N_{0}$ are constants that represent the average transmission energy per bit and noise power density of the additive white Gaussian noise channel. We assume binary phase shift keying modulation which is widely used in acoustic modems [23]. In accordance with [24], the bit error probability over distance $d$ can then be expressed as,

$$
q_{e}(d)=\frac{1}{2}\left(1-\sqrt{\frac{\Gamma(d)}{1+\Gamma(d)}}\right) .
$$

For a data packet with $m$ bits, the probability that the packet is successfully delivered over a distance $d$ therefore equals,

$$
p_{m}(d)=\left(1-q_{e}(d)\right)^{m} .
$$

For ease of notation, we introduce notation for the Euclidean distance between nodes. Let $d_{i j}^{i^{\prime} j^{\prime}}$ represent the 
distance between nodes $(i, j)$ and $\left(i^{\prime}, j^{\prime}\right)$,

$$
d_{i j}^{i^{\prime} j^{\prime}}=\sqrt{\left(\frac{\Delta_{D}}{L}\right)^{2}\left(i-i^{\prime}\right)^{2}+\left(\frac{\Delta_{W}}{M}\right)^{2}\left(j-j^{\prime}\right)^{2}} .
$$

In addition, let $N^{i j}=\left\{\left(i^{\prime}, j^{\prime}\right): d_{i j}^{i^{\prime} j^{\prime}} \leq T_{r}\right\}$ be the set of nodes within transmission range of node $(i, j)$.

In DBR, a packet is accepted by a node at position $\left(i^{\prime}, j^{\prime}\right)$ if (i) node $\left(i^{\prime}, j^{\prime}\right)$ is in the transmission range of the sender node $(i, j)$, (ii) node $\left(i^{\prime}, j^{\prime}\right)$ successfully receives the packet and (iii) all the nodes located in $N^{i j}$ that are above $\left(i^{\prime}, j^{\prime}\right)$ do not accept the packet. Let $P_{i j}^{i^{\prime} j^{\prime}}$ denote the probability that a data packet is successfully received at $\left(i^{\prime}, j^{\prime}\right)$ when sent from $(i, j)$, we then have,

$$
P_{i j}^{i^{\prime} j^{\prime}}=b_{j^{\prime}} p_{m}\left(d_{i j}^{i^{\prime} j^{\prime}}\right) \prod_{\ell=i^{\prime}+1}^{L}(1-Q(\ell ; i, j)),
$$

for $\left(i^{\prime}, j^{\prime}\right) \in N^{i j}$ and where $Q(\ell ; i, j)$ is the probability that the packet is successfully delivered at level $\ell$,

$$
Q(\ell ; i, j)=\sum_{k=0}^{M} b_{k} p_{m}\left(d_{i j}^{\ell k}\right) 1_{\left\{(\ell, k) \in N^{i j}\right\}} .
$$

Here $1_{\{\cdot\}}$ denotes the indicator function which equals 1 if its argument is true, and 0 if its argument is false. In other words, the sum above only includes nodes within the transmission range of the node at position $(i, j)$.

\subsection{Analysis of $k$-hop communication}

We now focus on the of number of hops needed for the data to be successfully delivered from source to sink. We first calculate the probability that any node in the network accepts the data packet from the source in one hop. Clearly nodes situated at an immediate upper level of the source will have zero probability to receive the data in one or more hops, as only direct communication is possible. Let $S$ be the horizontal position of the source at the bottom level and $T$ be the horizontal position of the sink at the surface level of the network. Let $h_{k}(i, j)$ represent the probability that the data is accepted by node $(i, j)$ in $k$ hops. The one-hop probability can be written as,

$$
h_{1}(i, j)=\sum_{i^{\prime}=1}^{i-1} \sum_{j^{\prime}=1}^{M} P_{i^{\prime} j^{\prime}}^{i j} P_{0 S}^{i^{\prime} j^{\prime}} .
$$

Note that the terms in the sum are only non-zero for $\left(i^{\prime}, j^{\prime}\right) \in N^{0 S} \cup N^{i j}$.

Similarly, the delivery probability in two hops can be written as:

$$
h_{2}(i, j)=\sum_{i^{\prime}=1}^{i-1} \sum_{j^{\prime}=1}^{M} P_{i^{\prime} j^{\prime}}^{i j} h_{1}\left(i^{\prime}, j^{\prime}\right),
$$

while the delivery probability of $k$ hop communication can be written by induction as,

$$
h_{k}(i, j)=\sum_{i^{\prime}=1}^{i-1} \sum_{j^{\prime}=1}^{M} P_{i^{\prime} j^{\prime}}^{i j} h_{k-1}\left(i^{\prime}, j^{\prime}\right) .
$$

Given the values $h_{k}(i, j)$, we can now easily express the delivery probability at the sink. Indeed, the packet reaches the sink if it is delivered in any number of hops,

$$
\bar{h}=\sum_{k=0}^{L} h_{k}(L, T) \text {. }
$$

For these calculations as well as for the delay and energy consumption calculations below, we implicitly make the simplifying assumption that the position of the nodes at the different levels is independent from hop to hop. This is indeed an approximation: the acceptance at a certain level depends on the position of the nodes at higher levels, as it is more probable to accept at a level if nodes at higher levels are at a larger horizontal distance from the transmitter. While we retain the position of the node that accepted the packet, we resample the positions of the nodes at higher levels. We will verify by simulation that this simplification does not void our results in section 5 .

\subsection{Computation of the mean end-to-end delay}

The propagation speed of the acoustic signal in water is $v_{0}=1500 \mathrm{~m} / \mathrm{s}$ which is much lower than that of terrestrial radio-frequency based signals. Thus, the propagation delay is significant in UWSN and can have a considerable impact on the performance of the system. Apart from the propagation delay, we also need to account for the effect of holding times which depend on the depth difference between sending and receiving nodes. Let $D_{P}\left(i, j ; i^{\prime}, j^{\prime}\right)$ and $D_{H}\left(i, j ; i^{\prime}, j^{\prime}\right)$ denote the propagation and holding time delays when the packet is sent from $(i, j)$ to $\left(i^{\prime}, j^{\prime}\right)$, then,

$$
D_{P}\left(i, j ; i^{\prime}, j^{\prime}\right)=\frac{d_{i j}^{i^{\prime} j^{\prime}}}{v_{0}}
$$

and,

$$
D_{H}\left(i, j ; i^{\prime}, j^{\prime}\right)=\frac{2 \tau}{\delta}\left(T_{r}-\frac{\Delta_{D}}{L}\left(i^{\prime}-i\right)\right),
$$

for $i^{\prime}>i$ and $\left(i^{\prime}, j^{\prime}\right) \neq(L, T)$. Furthermore, $D_{H}(i, j ; L, T)=$ 0 , as there is no holding time at the sink.

In order to find the mean delay, each possible path of the network needs to be explored. To this end, let $W_{i, j}$ denote the waiting time for a packet to reach $(i, j)$ and let $H_{i, j}$ denote the number of hops it takes. We now calculate the mean delays $w_{i j}^{k}$, given that it takes $k$ hops to reach $(i, j)$,

$$
w_{i j}^{k}=\mathrm{E}\left[W_{i, j} 1_{\left\{H_{i, j}=k\right\}}\right] \text {. }
$$

We again use a recursive scheme, similar to that for the delay calculations, for calculating the acceptance probability for the data packets in $k$ hops. To start, we find the delay for one hop communication,

$$
\begin{aligned}
w_{i j}^{1}=\sum_{i^{\prime}=1}^{i-1} \sum_{j^{\prime}=0}^{M} & \left(D_{H}\left(0, S ; i^{\prime}, j^{\prime}\right)+D_{P}\left(0, S ; i^{\prime}, j^{\prime}\right)\right. \\
& \left.+D_{H}\left(i^{\prime}, j^{\prime} ; i, j\right)+D_{P}\left(i, j ; i^{\prime}, j^{\prime}\right)\right) P_{0 S}^{i^{\prime} j^{\prime}} P_{i^{\prime} j^{\prime}}^{i j}
\end{aligned}
$$


To calculate $w_{i j}^{k}$ for $k>1$, we condition on the position of the last hop. That is, to reach $(i, j)$ in $k$ hops, we need to reach some $\left(i^{\prime}, j^{\prime}\right)$ in $k-1$ hops, and then reach $(i, j)$ by direct communication. The waiting time is then the sum of the waiting time to reach $\left(i^{\prime}, j^{\prime}\right)$ and the transmission and holding times to reach $(i, j)$ from $\left(i^{\prime}, j^{\prime}\right)$,

$$
\begin{aligned}
w_{i j}^{k}=\sum_{i^{\prime}=1}^{i-1} \sum_{j^{\prime}=0}^{M}\left(w_{i^{\prime} j^{\prime}}^{k-1}+\right. & D_{H}\left(i^{\prime}, j^{\prime} ; i, j\right) h_{k-1}\left(i^{\prime}, j^{\prime}\right) \\
& \left.+D_{P}\left(i^{\prime}, j^{\prime} ; i, j\right) h_{k-1}\left(i^{\prime}, j^{\prime}\right)\right) P_{i^{\prime} j^{\prime}}^{i j}
\end{aligned}
$$

Finally, we can calculate the mean end-to-end waiting time, conditional on the packet reaching the sink, by summing over the number of hops that it takes to reach $(L, T)$, and by dividing by the probability that the packet reaches the sink,

$$
\bar{W}=\frac{1}{\bar{h}} \sum_{k=0}^{L} w_{L T}^{k}
$$

\subsection{Energy consumption}

In order to study the expected energy consumption in the network, we recursively calculate the energy consumption for all node positions in the network to transmit to a particular node in a fixed number of hops. More precisely, let $C_{i j}\left(i^{\prime}, j^{\prime}\right)$ denote the energy consumption in position $\left(i^{\prime}, j^{\prime}\right)$ for transmitting from the source to node $(i, j)$ and let $H_{i . j}$ denote the number of hops to transmit to node $(i, j)$ as before, we then study the mean energy consumption given the number of hops,

$$
c_{i j}^{k}\left(i^{\prime}, j^{\prime}\right)=\mathrm{E}\left[C_{i j}\left(i^{\prime}, j^{\prime}\right) 1_{\left\{H_{i, j}=k\right\}}\right] .
$$

Note that $c_{i j}^{k}\left(i^{\prime}, j^{\prime}\right)=0$ for $i \leq i^{\prime}$ since nodes above $(i, j)$ cannot forward to $(i, j)$.

To start with one hop communication, we have

$$
c_{i j}^{1}\left(i^{\prime}, j^{\prime}\right)=\gamma P_{i^{\prime} j^{\prime}}^{i j} P_{0 S}^{i^{\prime} j^{\prime}} .
$$

Here $\gamma$ denotes the amount of energy a single transmission takes. That is, $C_{i j}\left(i^{\prime}, j^{\prime}\right)=\gamma$ if the single forwarding hop is in position $\left(i^{\prime}, j^{\prime}\right)$ and $C_{i j}\left(i^{\prime}, j^{\prime}\right)=0$ if this is not the case.

We further calculate the values $c_{i j}^{k}\left(i^{\prime}, j^{\prime}\right)$ for $k>0$ recursively. There is energy consumption at position $\left(i^{\prime}, j^{\prime}\right)$ if it is reached in $k-1$ hops, followed by direct communication, or if $\left(i^{\prime}, j^{\prime}\right)$ is part of a $(k-1)$-hop path to some intermediate node (above level $i^{\prime}$ ), from which $(i, j)$ is reached. We have,

$$
c_{i j}^{k}\left(i^{\prime}, j^{\prime}\right)=\gamma h_{k}\left(i^{\prime}, j^{\prime}\right) P_{i^{\prime} j^{\prime}}^{i j}+\sum_{\ell=i^{\prime}+1}^{i-1} \sum_{m=0}^{M} c_{\ell m}^{k-1}\left(i^{\prime}, j^{\prime}\right) P_{\ell m}^{i j} .
$$

We can finally calculate the energy consumption at each level in the network, by summing over the number of hops to reach the destination, and by summing over the different positions at the same level,

$$
c(i)=\sum_{k=0}^{L} \sum_{m=0}^{M} c_{L T}^{k}(i, m)
$$

\subsection{Extension to 3 dimensions}

We briefly discuss how the model can be extended to 3 dimensions (3D). We still consider an UWSN with $L+1$ nodes with sink at the surface and source at the bottom. While assuming fixed positions for source and sink, we allow for movement of the nodes that relay the information. In particular, we divide the total depth difference $\Delta_{D}$ between source and sink into $L+1$ depth levels, level 0 being the level of the source and level $L$ being the level of the sink.

At each horizontal level, the node can be in $M$ positions. As the horizontal level is a plane, there are e.g. $M$ positions on a square grid, or $M$ discrete positions evenly distributed on a disk. The distance calculations are the key difference between the 2D and 3D model. For ease of notation, we introduce a common coordinate system on all levels, and let $(x(j), y(j))$ denote the (horizontal) coordinates of position $j$. The distance between node $j$ on level $i$ and node $j^{\prime}$ on level $i^{\prime}$ can then be expressed as follows,

$$
d_{i j}^{i^{\prime} j^{\prime}}=\sqrt{\left(\frac{\Delta_{D}}{L}\right)^{2}\left(i-i^{\prime}\right)^{2}+\left(x(j)-x\left(j^{\prime}\right)\right)^{2}+\left(y(j)-y\left(j^{\prime}\right)\right)^{2}} .
$$

Given this modification of the distance calculations, the remainder of the calculations remain valid. Note however that the summations in sections 4.3 till 4.6 now run over all positions in a horizontal plane.

\section{Numerical Results}

In this section, we numerically study different performance measures of the model at hand. We assume that the total depth of the network is $\Delta_{D}=500 \mathrm{~m}$ with $L=50$ depth levels. Note that there is only one sensor node at each depth level meaning there are 50 nodes in the network. We keep the values of the numerical parameters in line with the values in [2]: we set $s=2, v_{0}=1500 \mathrm{~m} / \mathrm{s}$, $\delta=T_{r} / 4$ and $\tau=T_{r} / v_{0}$. Finally, we assume data packets of 50 bytes. These parameter values are used in all plots.

\subsection{Delivery probabilities}

We first investigate the delivery probabilities for direct communication. The source is fixed at bottom level of the network at horizontal position $S$. We assume that each node can move horizontally to $M=50$ locations over a range of $\Delta_{W}=500 \mathrm{~m}$, each horizontal position being equally likely. We further choose the transmission energy such that $e_{b} / N_{0}=57 \mathrm{~dB}$, and assume that the transmission range is only bounded by transmission errors $\left(T_{r}=\infty\right)$.

Fig. 2(a) shows the delivery probability by a direct transmission from the source to a node at level $10 \mathrm{vs}$. the horizontal position of this node for different source positions $S$ as indicated. As expected, the maximal delivery probability is obtained when source and destination are aligned, as this corresponds to the shortest distance between these nodes. 


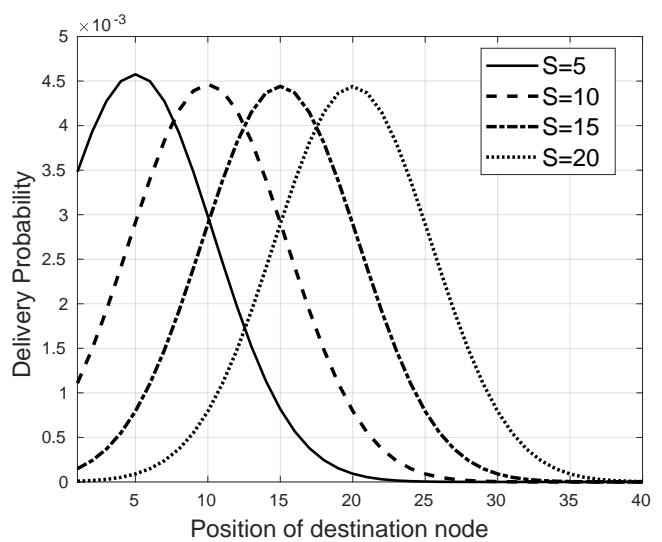

(a)

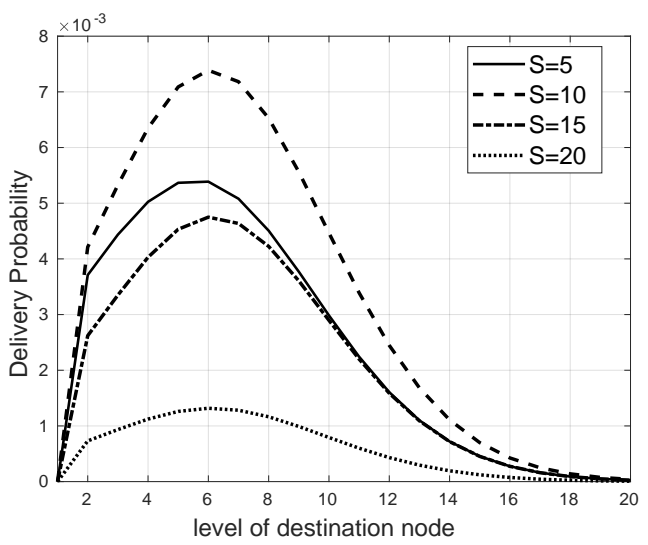

(b)

Figure 2: Delivery probability for direct transmission from source to sink (a) for different horizontal positions of the destination and (b) for different levels of destination

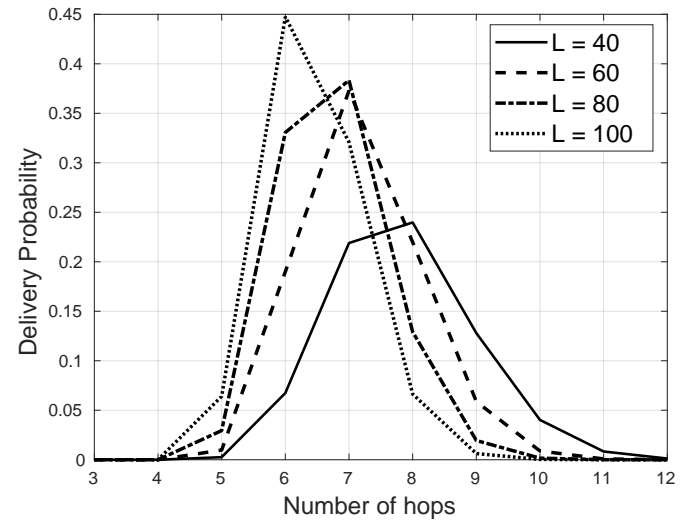

(a)

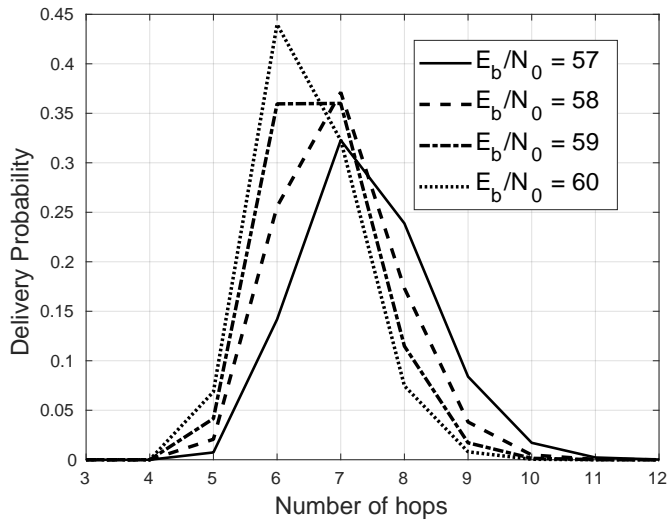

(b)

Figure 3: Delivery probability (a) for different total number of depth levels and (b) for different $e_{b} / N_{0}$

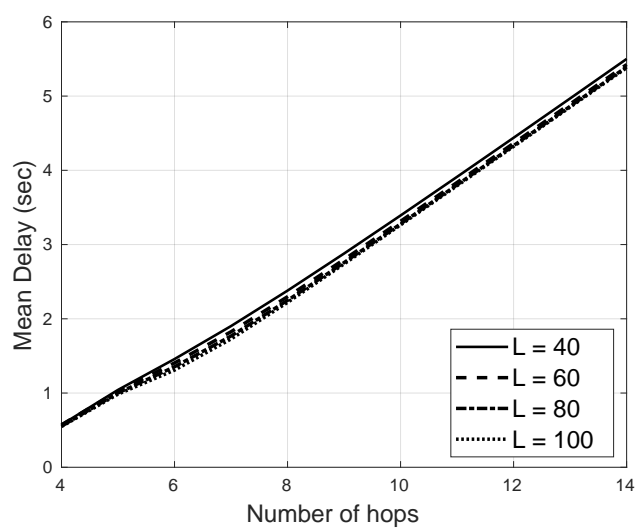

(a)

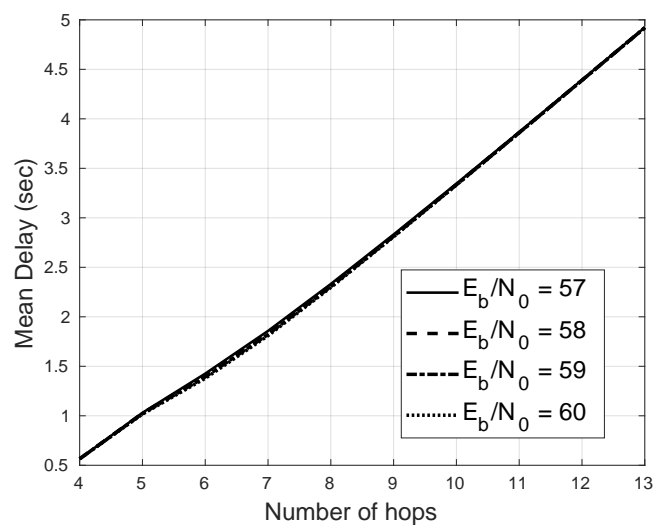

(b)

Figure 4: Mean Delay (a) for different total number of depth levels and (b) for different $e_{b} / N_{0}$ 


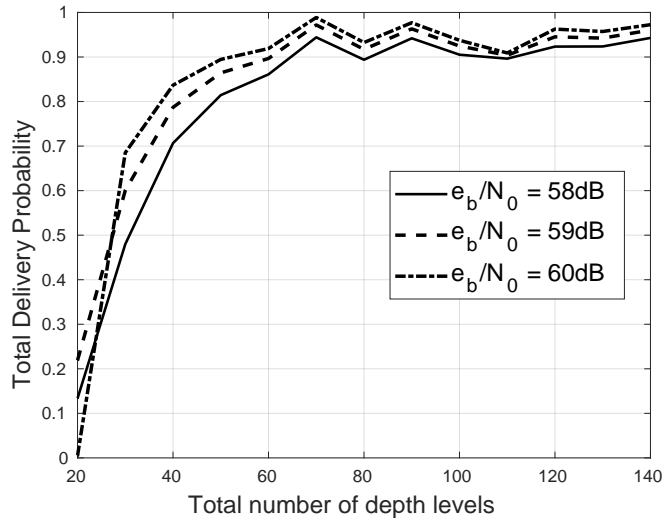

(a)

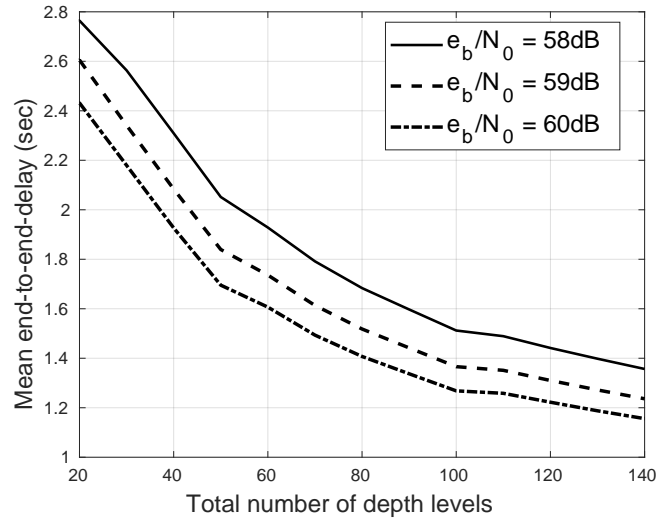

(b)

Figure 5: Total delivery probability and mean end-to-end delay

Fig. 2(b) shows the same delivery probability, but we now fix the horizontal position of the destination node at 10 and vary its depth level. Again, different source positions $S$ are assumed as indicated. There is an outspoken optimal depth level: at first the delivery probability is small as it is likely that the packet will be delivered to higher nodes. The delivery probability therefore first increases with the depth level. However, if the depth level is already high, the chance to successfully transmit is low. Hence, the delivery probability decreases with increasing depth levels.

For the remaining plots, we assume that the node can move into $M=15$ slots spanning a range $\Delta_{W}=150 \mathrm{~m}$. We set the transmission range to one-fifth of the total range of the network i.e., $T_{r}=\Delta_{D} / 5=100 \mathrm{~m}$ and fix the horizontal position of the source and sink: $S=T=10$.

We now focus on the number of hops needed for the data to be delivered at the sink. Note that the number of hops cannot exceed the number of depth levels as no downward transmissions are allowed in DBR. Fig. 3(a) and Fig 3(b) depict the probability mass function of the number of hops needed to deliver the packet from source to sink. In 3(a), we depict the probability mass function for networks with different levels $L$. In 3(a), we depict the probability mass function for different transmission powers $e_{b} / N_{0}$.

From the figures, we can observe that the delivery probability is zero for the first 4 hops which is not surprising as the transmission range is $100 \mathrm{~m}$. Moreover, most of the probability mass is between 5 and 9 hops, which is again in line with the transmission range. Finally, it is seen that end-to-end communication with fewer hops is more likely if there are more nodes (i.e., by increasing $L$, see Fig. 3(a)), or if the transmission power increases (see Fig. 3(b)).

\subsection{Mean end-to-end delay}

Fig. 4 depicts the mean delay conditioned on the number of hops required for the data to reach the sink. Figure 4(a) fixes $e_{b} / N_{0}$ to $57 \mathrm{~dB}$ and shows the mean delay for different numbers of depth levels $L$ between the source and the sink. On the other hand Fig. 4(b) fixes the number of depth levels to $L=50$ and varies the transmission power as indicated. It is readily seen that the the conditional end-to-end delay grows almost linearly with the number of hops, and is largely insensitive to changes in transmission power and the number of depth levels. This is not entirely unexpected as the end-to-end delay is largely dominated by the holding times at the nodes.

In Fig. 5, we depict the delivery probability at the sink and the corresponding mean end-to-end delay (conditional on the packet reaching the sink). We again set the total depth to $\Delta_{D}=500 \mathrm{~m}$ and vary the number of depth levels. As we fix the total depth, increasing the number of levels means that the depth difference between adjacent levels decreases. Fig. 5(a) depicts the delivery probability at the sink vs. the number of depth levels for different $e_{b} / N_{0}$ as indicated. It can be seen from the figure that the total delivery probability in general increases when the number of depth levels increases. This is expected as we add additional sensors, making it less likely that the packet is lost. The curve is not monotone increasing though, which can be explained by the interplay between the depth levels and the fixed transmission range $T_{r}$. Fig. $5(\mathrm{~b})$ shows that the mean end-to-end delay decreases when the number of depth levels increases. Moreover, while the delivery probability increases with $e_{b} / N_{0}$, it has the opposite effect on the mean end-to-end delay.

In Fig. 6(a), we study the effect of the scaling parameter $\delta$ on the mean delay. The parameter $\delta$ is key for the holding times. Larger $\delta$ implies shorter holding times at each intermediate node and thus reduces the mean delay. Fig. 6(a) depicts the mean end-to-end delay, conditional on the number of hops. As previously noted, the end-toend delay grows approximately linearly with the number of hops. It can now clearly be seen that the slope of the 


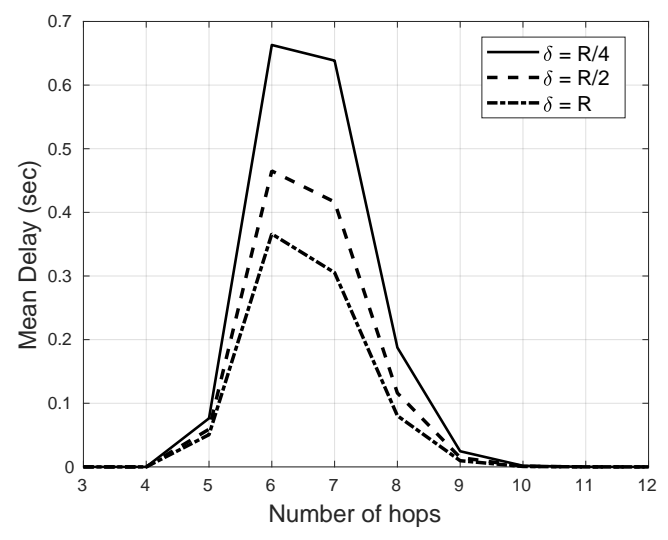

(a)

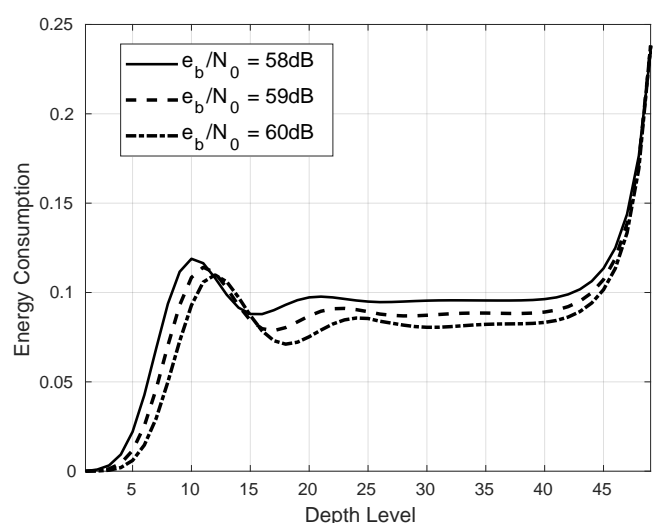

(b)

Figure 6: Mean Delay and Energy consumption

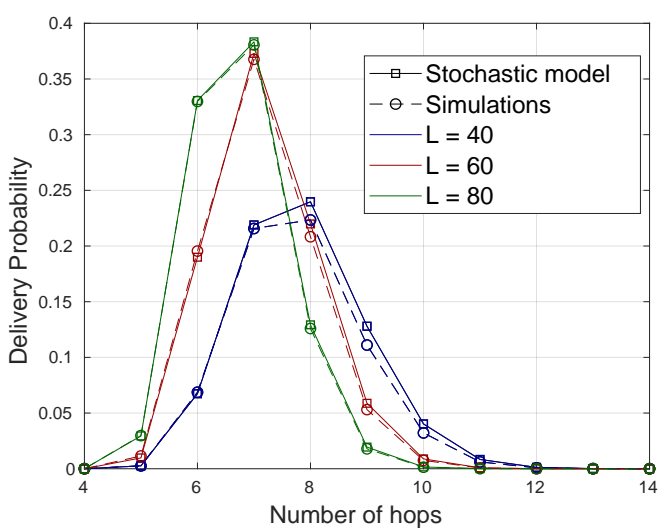

(a)

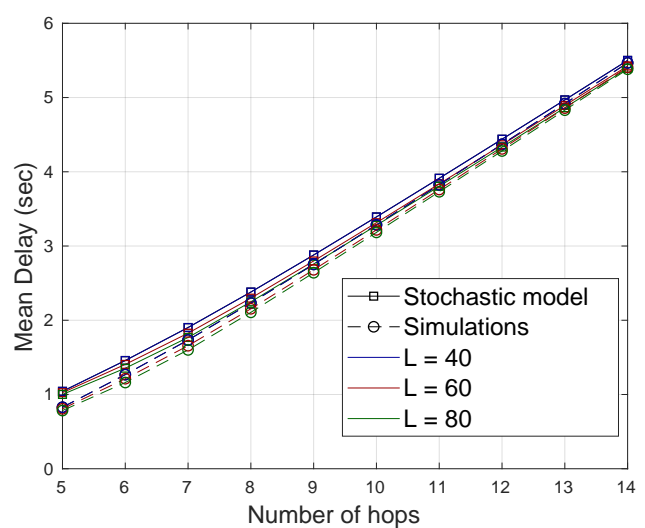

(b)

Figure 7: Comparison of stochastic model and Monte-Carlo simulation for hops distribution and Mean delay

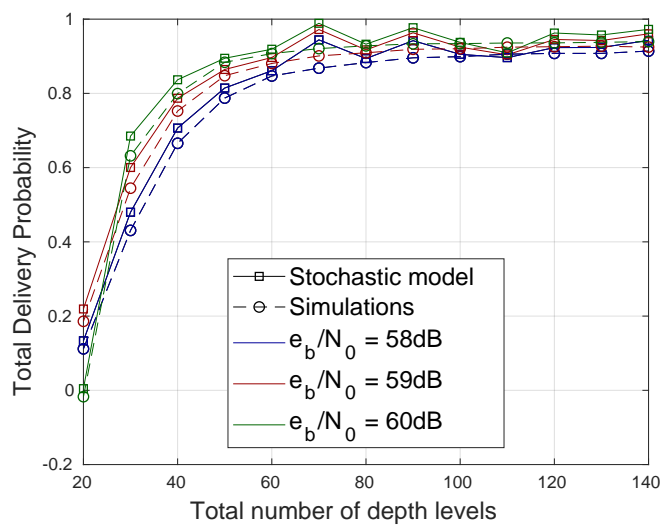

(a)

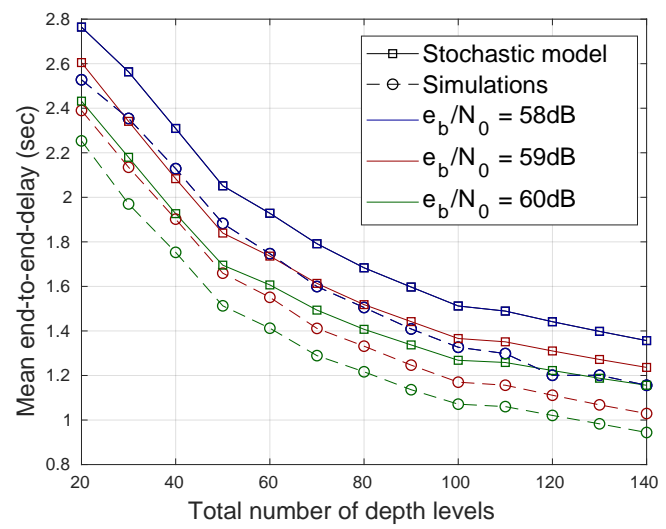

(b)

Figure 8: Comparison of stochastic model and Monte-Carlo simulation for Total delivery probability and Mean end-to-end delay 
conditional end-to-end delay depends on $\delta$.

\subsection{Energy consumption}

Finally, Fig. 6(b) depicts the energy consumption at the different depth levels of the network. We assume that the amount of energy for one transmission is $\gamma=1$, such that the plot depicts the mean number of times the nodes at each level participate in the transmission. Ideally, one aims for uniform energy expenditure such that the life time of all nodes is approximately equal. It is however clear from the figure that the energy consumption at levels close to both source and sink is higher, compared to the energy consumption by nodes in the middle of the network. This suggests that it is beneficial to increase the density of the nodes near sink and source.

\subsection{Model validation by simulation}

We now evaluate the performance of the model through simulations and compare its time complexity with the stochastic model.

Recall that the analytical model made the following simplifying assumption. The position of the nodes above a transmitting node, is assumed independent of their positions during preceding transmissions. In reality, these positions may largely remain the same, and therefore we need to verify that this assumption does not compromise the accuracy of our results. To this end, we compare our results with results obtained by Monte Carlo simulation, where it is assumed that the positions of the nodes do not change throughout the multi-hop transmission of the packet.

In particular, the details of the simulation study are as follows. We consider $K$ replications of a multi-hop transmission from source to sink. For each replication, we first randomly draw the positions of the nodes at all levels. We then calculate the performance measures using the analytical model of section 4, for the given fixed node positions. As the positions of the nodes are fixed, the calculations of section 4 are exact. Each replication yielding values for the various performance measures, we finally calculate the sample averages, and corresponding confidence intervals for these performance measures.

Analytic and simulation results are numerically compared in Figs. 7 and 8. We keep the same size of the network: a depth of $500 \mathrm{~m}$ and a width of $150 \mathrm{~m}$. The number of available positions that a node can take at a particular depth level are fixed to $M=15$ in accordance with the stochastic model. We sample $K=1000$ times, which is sufficient to obtain a confidence $95 \%$ confidence interval of $\pm 0.5 \%$ the sample mean. Although the source and sink can be randomly located at bottom and surface level of the network respectively, we place them both at a position 10 for consistency in the experiment. All the other network parameters are similar to those in the stochastic model.

Fig. 7(a) depicts the comparison of the number of hops distribution where Fig. 7(b) shows the comparison of the mean delay for different number of deployed nodes in the network. Furthermore, fig. 8(a) depicts the the total delivery probability and fig. 8(b) shows the mean end-to-end delay for both models. It can be seen from the figure that the difference between the results obtained by simulation and analytically is negligible.

To compare the efficiency of the analytical model and the simulation model, we compare their time complexity. For the stochastic model, most of the time is spent on the calculation of the delivery probability and hops distributions. In the worst case scenario, the time complexity of the stochastic model is $O\left(L^{3} M^{2}\right)$, where $L$ is the number of depth levels in the network and $M$ is the number of positions that a node can take horizontally. For the simulation study, we only sample the positions of the nodes, and then calculate the performance measures by the analytic approach to reduce the variance of the Monte-Carlo simulation. This technique is referred to as variance reduction by conditioning, see e.g. [25]. Hence, the time complexity of a single iteration is $O\left(L^{3}\right)$, which corresponds to the complexity of the stochastic model with $M=1$ (as the nodes are at a fixed position in each iteration). For moderate $M$, the number of samples $K$ that are needed in the Monte Carlo simulations considerably exceeds $M^{2}$, which implies that the stochastic model can calculate the various performance measures faster, even after applying the variance reduction technique (which also relies on the model).

\section{Conclusion}

In this paper, we have proposed a numerically tractable stochastic model for the performance evaluation of DBR. Specifically, we have considered four performance metrics: the hop-distribution, the packet delivery probability, the expected energy consumption and the expected end-to-end delay. The model has been validated by comparing the average performance indices obtained by its analysis with the estimates obtained by a stochastic simulation. By a numerical example, we have illustrated that our model can be used to assess the impact of various network configuration parameters (e.g., the transmission power and the scaling factor $\delta$ defined by DBR) on these indices. Our analysis showed that the number of hops in the route can dramatically affect the performance of the protocol. The proposed model can be further used for optimization purposes given the limited computational effort required, in comparison to underwater sensor network simulations.

\section{References}

[1] R. W. Coutinho, A. Boukerche, L. F. Vieira, A. A. Loureiro, Design guidelines for opportunistic routing in underwater networks, IEEE Communications Magazine 54 (2) (2016) 40-48.

[2] H. Yan, Z. J. Shi, J.-H. Cui, DBR: Depth-based routing for underwater sensor networks, in: Proceedings of NETWORKING 2008: Ad Hoc and Sensor Networks, Wireless Networks, Next 
Generation Internet. Lecture Notes in Computer Science, vol 4982, 2008, pp. 72-86.

[3] G. Tuna, V. C. Gungor, A survey on deployment techniques, localization algorithms, and research challenges for underwater acoustic sensor networks, International Journal of Communication Systems 30 (17) (2017) e3350.

[4] Z. Guan, T. Melodia, D. Yuan, Stochastic channel access for underwater acoustic networks with spatial and temporal interference uncertainty, in: Proceedings of the Seventh International Conference on Underwater Networks \& Systems, WUWNet, ACM, 2012, pp. 18:1-18:8.

[5] F. Pignieri, F. De Rango, F. Veltri, S. Marano, Markovian approach to model underwater acoustic channel: Techniques comparison, in: Proceedings of the IEEE Conference on Military Communications MILCOM 2008, IEEE, 2008, pp. 1-7.

[6] Z. Zhou, Z. Peng, J.-H. Cui, Z. Shi, Efficient multipath communication for time-critical applications in underwater acoustic sensor networks, IEEE/ACM Transactions on Networking (TON) 19 (1) (2011) 28-41.

[7] M. Faheem, G. Tuna, V. C. Gungor, Lrp: Link quality-aware queue-based spectral clustering routing protocol for underwater acoustic sensor networks, International Journal of Communication Systems 30 (12).

[8] A. Stefanov, M. Stojanovic, Design and performance analysis of underwater acoustic networks, IEEE Journal on Selected Areas in Communications 29 (10) (2011) 2012-2021.

[9] F. A. de Souza, B. S. Chang, G. Brante, R. D. Souza, M. E. Pellenz, F. Rosas, Optimizing the number of hops and retransmissions for energy efficient multi-hop underwater acoustic communications, IEEE Sensors Journal 16 (10) (2016) 3927-3938.

[10] D. Marinakis, K. Wu, N. Ye, S. Whitesides, Network optimization for lightweight stochastic scheduling in underwater sensor networks, IEEE Transactions on Wireless Communications 11 (8) (2012) 2786-2795.

[11] L. M. Brekhovskikh, Lysanov, Fundamentals of ocean acoustics, Springer, 2003.

[12] D. Li, J. Du, L. Liu, A data routing algorithm based on markov model in underwater wireless sensor networks, in: Proceedings of the IEEE International Conference on Ubiquitous Wireless Broadband (ICUWB), 2016, IEEE, 2016, pp. 1-4.

[13] M. Jafri, A. Marin, A. Torsello, M. Ghaderi, On the optimality of opportunistic routing protocols for underwater sensor networks, in: In Proc. of MSWiM, 2018, pp. 207-215.

[14] H. Yu, N. Yao, T. Wang, G. Li, Z. Gao, G. Tan, Wdfad-dbr Weighting depth and forwarding area division dbr routing protocol for uasns, Ad Hoc Networks 37 (2016) 256-282.

[15] M. A. Rahman, Y. Lee, I. Koo, Eecor: an energy-efficient cooperative opportunistic routing protocol for underwater acoustic sensor networks, IEEE Access 5 (2017) 14119-14132.

[16] C.-M. Chao, C.-H. Jiang, W.-C. Li, Drp: An energy-efficient routing protocol for underwater sensor networks, International Journal of Communication Systems, 201730 (15).

[17] S. Basagni, C. Petrioli, R. Petroccia, D. Spaccini, Carp: A channel-aware routing protocol for underwater acoustic wireless networks, Ad Hoc Networks 34 (2015) 92-104.

[18] P. Xie, Z. Zhou, Z. Peng, J.-H. Cui, Z. Shi, Sdrt: A reliable data transport protocol for underwater sensor networks, Ad Hoc Networks 8 (7) (2010) 708-722.

[19] N. Nowsheen, G. Karmakar, J. Kamruzzaman, Pradd: A path reliability-aware data delivery protocol for underwater acoustic sensor networks, Journal of Network and Computer Applications 75 (2016) 385-397.

[20] M. R. Jafri, S. Ahmed, N. Javaid, Z. Ahmad, R. Qureshi, Amctd: Adaptive mobility of courier nodes in threshold-optimized dbr protocol for underwater wireless sensor networks, in: Proceedings of Eighth International Conference on Broadband and Wireless Computing, Communication and Applications (BWCCA), 2013, IEEE, 2013, pp. 93-99.

[21] M. Stojanovic, On the relationship between capacity and distance in an underwater acoustic communication channel, ACM SIGMOBILE Mobile Computing and Communications Review
11 (4) (2007) 34-43.

[22] P. S. Duke, Direct-sequence spread-spectrum modulation for utility packet transmission in underwater acoustic communication networks, Tech. rep., Naval Postgraduate school Monterey CA, USA (2002).

[23] L. Freitag, M. Grund, S. Singh, J. Partan, P. Koski, K. Ball, The whoi micro-modem: an acoustic communications and navigation system for multiple platforms, in: Proceedings of Oceans 2005 MTS/IEEE, 2005, pp. 1086-1092 Vol. 2.

[24] Y. Noh, U. Lee, P. Wang, B. S. C. Choi, M. Gerla, Vapr: voidaware pressure routing for underwater sensor networks, IEEE Transactions on Mobile Computing 12 (5) (2013) 895-908.

[25] S. Asmussen, P. Glynn, Stochastic Simulation: Algorithms and Analysis, Springer, 2007 